\title{
Temperature and Pressure Dependences of the Grüneisen Constant of a Mat of Polyethylene Single Crystals
}

\author{
Tsuyoshi KiJima, Kazunori Koga, Kiyohisa Imada, \\ and Motowo TAKAYANAGI \\ Department of Applied Chemistry, Faculty of Engineering, \\ Kyushu University, Fukuoka, Japan.
}

(Received April 5, 1974)

\begin{abstract}
The sound velocity of the longitudinal ultrasonic wave at $4.64 \mathrm{MHz}$ in a polyethylene (PE) single crystal mat was measured at various temperatures from $15^{\circ} \mathrm{C}$ up to $90^{\circ} \mathrm{C}$ and at various pressures below $5500 \mathrm{~atm}$. The Grüneisen constant, $\gamma_{\mathrm{s}}$, for PE single crystals was evaluated from the pressure dependence of the sound velocity. The $\gamma_{\mathrm{s}}$ values at $1 \mathrm{~atm}$ increased with increasing temperature and showed larger values than those for the perfect crystals predicted by the theory proposed by Broadhurst and Mopsik. This fact was discussed in connection with the mean square displacements of molecules in PE crystals obtained by X-ray measurements by Iohara, et al. The increase of the Grüneisen constant with increasing temperature was explained by an increase of molecular displacements in the crystal due to lattice imperfections as well as to the anharmonicity of intermolecular vibrations. The $\gamma_{\mathrm{s}}$ value at higher pressures showed smaller dependence on temperature. This fact was explained in terms of decreases in the volume of lattice imperfections and in anharmonicity of intermolecular potential with increasing pressure which are probably accelerated by compression of the crystal lattice.
\end{abstract}

KEY WORDS Ultrasonics / High Pressure / Grüneisen Constant / Anharmonicity / Single Crystal / Polyethylene /

This paper is concerned with the temperature and pressure dependences of the Grüneisen constant for polyethylene single crystals. The Grüneisen constant is a useful parameter to express the anharmonicity of molecular motions in a crystal lattice. The Grüneisen constant of polyethylene has been most extensively studied among other polymers. The experimental studies were made using various methods, such as infrared absorption, ${ }^{1,2}$ Raman scattering, ${ }^{3}$ compressibility, ${ }^{4,5}$ sound velocity, ${ }^{6}$ and thermal conductivity. ${ }^{2}$ On the other hand, Broadhurst and Mopsik $^{4,7}$ have derived theoretically the equation for the Grüneisen constant of polyethylene, which enables us to calculate the variation of it with volume or temperature. However, as was discussed in some detail by $\mathrm{Wu}$, Jura, and Shen, ${ }^{1}$ it should be emphasized that the values of the Grüneisen constant of polyethylene vary largely with its definitions and with the experimental techniques used in its determination. These are caused by the strong anisotropy of the crystal force field in that the methylene units are held by covalent bonds along the molecular axis but in the plane perpendicular to it by van der Waals forces. The Grüneisen constant defined based on the sound velocity, $\gamma_{\mathrm{s}}$, is considered to be related to the vibrational modes of molecules with lower frequency and thus it mainly represents the anharmonicity of intermolecular thermal vibrations in the polyethylene crystal lattice. ${ }^{6}$

In this study the temperature dependence of the $\gamma_{\mathrm{s}}$ value of polyethylene as well as the pressure effect on it was investigated over a temperature range above room temperature, where anharmonic molecular motions are expected to increase with increasing temperature. The $\gamma_{\mathrm{s}}$ values at different pressures were evaluated as a function of temperature above room temperature by ultrasonic measurements under various pressures. The anharmonicity of thermal vibrations of molecules at normal pressure was discussed by comparing the experimental values 
of $\gamma_{\mathrm{S}}$ with the theoretical values ${ }^{7}$ and by referring to the information on the mean square displacements of molecules in the crystal lattice evaluated by X-ray measurements by Iohara, et al., in our laboratories. ${ }^{8}$ The pressure effect on molecular motions in the crystal lattice was explained by referring to the pressure dependence of the $\gamma_{\mathrm{s}}$ values.

\section{EXPERIMENTAL}

\section{Sample Preparations}

The sample used in this study was a commercial high density linear polyethylene, Hizex $1200 \mathrm{~J}$, the product of Mitsui Petrochemical Industries Co., Ltd. Its molecular weight $\left(M_{v}\right)$ was $3.1 \times 10^{4}$.

Single crystals were prepared from a $0.05-\%$ xylene solution by isothermal crystallization at $80^{\circ} \mathrm{C}$, which was filtered to form an aggregate of crystal lamellae. The aggregate was compressed into a disk of $16-\mathrm{mm}$ diameter and 2 to $3-\mathrm{mm}$ thickness, the residual solvent being removed by evaporating under vacuum. The long period of the single crystal mat was evaluated by the small-angle X-ray method as $135 \AA$. The molecular chains in the mat were found to be oriented approximately perpendicular to the mat surface by the wide-angle X-ray scattering photograph.

\section{High Pressure Apparatus}

Figure 1 shows a schematic diagram of the high pressure vessel used in this study. The vessel is composed of double cylindrical layers. This was constructed by pressing the $0.35 \mathrm{~mm}$ oversized and tapered inner cylinder into the tapered outer one. Both of the inner and outer cylinders are made of SKD 11 steel and the diameter ratio of the outer to the inner cylinder is 2 . The vessel could be used up to a liquid pressure of $18000 \mathrm{~kg} / \mathrm{cm}^{2}$ according to calculations based on Manning's theory. ${ }^{9}$ At the top and bottom of the vessel plugs of hardened steel (SCM 3) with seals of copper, aluminum and lead were used. The bottom plug with a hole was connected with a pipe, which led via a pipe connector to the pressure intensifier. The manganine gauge for the pressure measurement was attached to the pipe connector. The top plug containing three couples of metal-sheathed wires

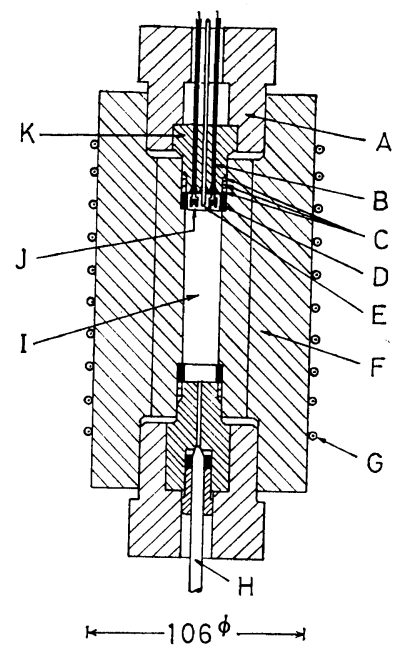

Figure 1. High pressure bomb: A, lock nut; B, sheathed wire; C, packings of copper, aluminum, and lead; D, screw box; E, thermocouple; F, double cylinder; G, sheathed heater; H, pipe; I, high pressure chamber; J, connector; K, electrode plug.

was used. The sheathes were welded to the plug by the same method as used by Hirakawa and Takemura. ${ }^{10}$ Silicone oil (TSF 431, the product of Toshiba Silicone Co.) was used as the pressure transmitting fluid. The pressure vessel was heated by the sheathed heater wound around the outside of the vessel.

\section{Ultrasonic Apparatus}

The ultrasonic measurements were conducted by the pulse method using solid aluminum buffers constructed in a similar manner to that reported by Hirakawa and Takemura. ${ }^{10}$ Figure 2 shows a schematic diagram of the measuring capsule, which was inserted in the high-pressure chamber filled with silicone oil. The disk-shaped sample was placed between two aluminum buffer rods and pressed by springs from both ends of the capsule. The X-cut quartz crystal was plastered at one side to each buffer rod by a conducting adhesive. Figure 3 shows a block diagram of the ultrasonic measurement. A rectanglar trigger pulse was generated by the pulse generator composed of astable and monostable multivibrators, and by feeding it to the ringing circuit a pulsed signal of radio frequency was generated. After amplifying the pulsed 


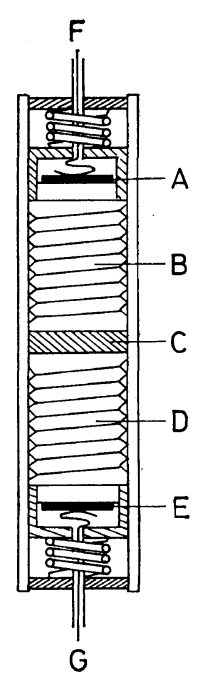

Figure 2. Schematic diagram of measuring capsule for the ultrasonic measurement: A, transmitting crystal; B, aluminum buffer; $\mathrm{C}$, sample; $D$, aluminum buffer; $E$, receiving crystal; $F$, input terminal; G, output terminal. The capsule is inserted in the high-pressure vessel and immersed in silicone oil.

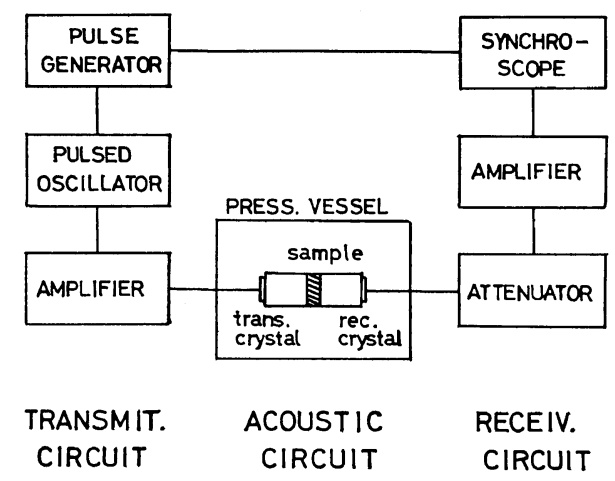

Figure 3. Block diagram of ultrasonic measurement.

radio frequency signal above a $p-p$ height of 100 volts, it was fed to the quartz ultrasonic pulse generator. The ultrasonic pulses propagate through the acoustic medium, which is composed of the sample sheet, aluminum buffers and very thin layers of silicone oil between them. The electric pulse retransduced by the quartz at the other end was fed via the attenuator and the amplifier into the synchroscope, the time axis of which was swept by the trigger pulse fed directly from the pulse generator.

\section{Experimental Procedure}

The disk of PE single crystals was precompressed for $2 \mathrm{hr}$ at $5000 \mathrm{~atm}$ and at room temperature in the high pressure chamber and then used for the subsequent ultrasonic measurement. During the precompression the disk exhibited a decrease in thickness of only 0.1 to $0.2 \%$. The measurement of the sound velocity under pressure was carried out with $4.64-\mathrm{MHz}$ longitudinal waves. After the interior of the pressure vessel including the ultrasonic capsule was filled with the silicone oil compressed to 4500 to $5500 \mathrm{~atm}$ at room temperature and then heated up to a predetermined temperature which was then fixed, the transmission time of the sound wave in the acoustic medium was measured at $200 \mathrm{~atm}$ intervals while the pressure was reduced stepwise. Such a procedure was conducted at various temperatures, i.e., $15,32,52,70$, and $90^{\circ} \mathrm{C}$ for the samples with different thicknesses. The sound velocity, $u$, was calculated from the data obtained on two samples of the same material but of different thicknesses using the equation

$$
u=\frac{d_{2}-d_{1}}{t_{2}-t_{1}}
$$

where $d_{1}$ and $d_{2}$ are the thicknesses of the samples 1 and 2, and $t_{1}$ and $t_{2}$ are the transmitting times measured when the samples 1 and 2 were used, respectively. The difference in thickness, $d_{2}-d_{1}$, was corrected using the $P-V-T$ data on polyethylene reported by Warfield. ${ }^{11}$

\section{RESULTS}

Figure 4 shows the pressure dependence of the sound velocity along the direction perpendicular to the plane surface of the polyethylene single crystal mat at various temperatures. Each curve shows the dispersion of the sound velocity in the pressure region above $2000 \mathrm{~atm}$, whereas no significant dispersion can be found below 2000 atm.

The Grüneisen constant, $\gamma_{s}$, can be evaluated using the pressure dependence of the sound velocity according to the relation of eq $2 .^{6}$ 


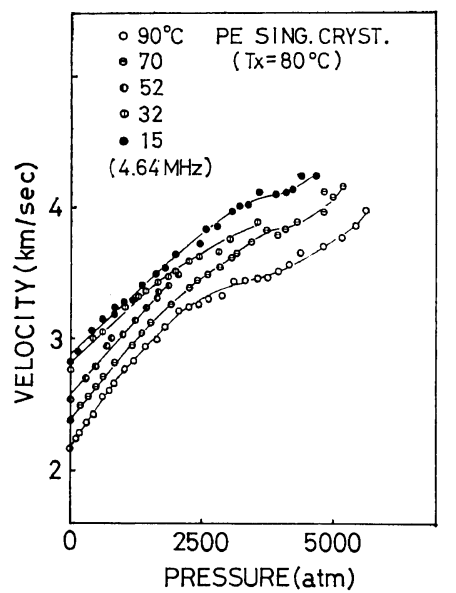

Figure 4. Pressure dependence of sound velocity for the single crystals of polyethylene at various temperatures.

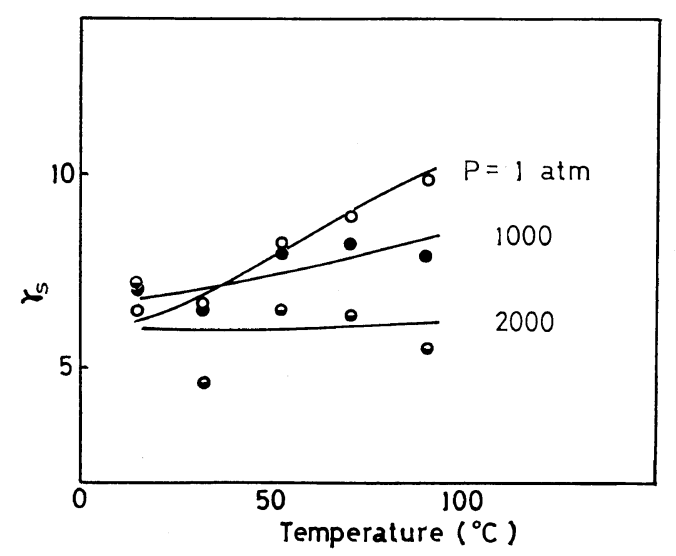

Figure 5. Temperature dependence of $\gamma_{\mathrm{s}}$ under 1, 1000 , and $2000 \mathrm{~atm}$.

$$
\gamma_{\mathrm{S}}=\left(\frac{\partial \ln u}{\partial P}\right)_{T} \frac{1}{\beta}+\frac{1}{3}
$$

where $u, \beta$, and $P$ are the sound velocity, compressibility, and pressure, respectively. In order to evaluate the $\gamma_{\mathrm{s}}$ for the polyethylene single crystal mat the sound velocity in the pressure region below $2000 \mathrm{~atm}$ in Figure 4 was approximated by a quadratic function of pressure, which was determined by the least-square method and then substituted into the first term of the right side of eq 2 . The $\beta$ value was evaluated using the $P-V-T$ data on the bulk sample of high-density polyethylene obtained by Hellwege, et al. ${ }^{12}$ As this sample has a very high crystallinity compared with that of the single crystal mat, this method will not induce a meaningful error. Figure 5 shows a plot of $\gamma_{\mathrm{s}}$ against temperature at 1,1000 , and $2000 \mathrm{~atm}$. The $\gamma_{\mathrm{s}}$ values at $1 \mathrm{~atm}$ increase remarkably with increasing temperature, but those at the higher pressures show smaller dependence on temperature.

\section{DISCUSSION}

\section{Implication of the Formulae for the Grüneisen Constants}

Since the Grüneisen constant defined by eq 2 is different from the originally defined one, it is necessary to follow the derivation of eq 2 and to confirm the assumption implicit in it.

The original definition of the Grüneisen constant, that is, the mode Grüneisen constant is given by

$$
\gamma_{j}=-\left(\frac{\partial \ln \nu_{j}}{\partial \ln V}\right)_{T}
$$

where $\nu_{j}$ is the frequency for the $j$-th vibrational mode and $V$ the volume. ${ }^{13}$ The mode Grüneisen constants for the optically active modes can be determined from eq 3 using the spectroscopic data. On the other hand, the Grüneisen constant for optically inactive or indetectable modes could be evaluated by using some approximate equations derived from eq 3 on the basis of some assumptions. $\mathrm{Eq} 2$ is one of those equations. From eq 3 the mode Grüneisen constant, $\gamma_{D}$, for the acoustic mode with Debye characteristic frequency, $\nu_{D}$, can be written by

$$
\gamma_{\mathrm{D}}=-\left(\frac{\partial \ln \nu_{\mathrm{D}}}{\partial \ln V}\right)_{T}
$$

The characteristic frequency, $\nu_{D}$, for three-dimensional Debye solid relates to the sound velocity on the basis of Debye's theory ${ }^{14}$ as follows:

$$
\nu_{\mathrm{D}} \simeq\left(\frac{3 N u^{3}}{4}\right)^{1 / 3}
$$

where $N$ is the number of vibrating units per unit volume of crystal.

Substitution of eq 5 into eq 4 results in

$$
\gamma_{\mathrm{D}} \simeq\left(\frac{\partial \ln u}{\partial P}\right)_{T} \frac{1}{\beta}+\frac{1}{3}
$$


Eq 2 used in the previous section is identical with the right side of eq 6 .

Further, according to Dugdale, et al. ${ }^{15}$ the sound velocity at pressure $P$ is related to the bulk modulus, $B$, by

$$
u \propto\{(B-P / 2) V\}^{1 / 2}
$$

By substituting eq 7 into eq 6 and then considering that the value of $P / B$ is negligibly small compared with unity, eq 8 is obtained. ${ }^{4}$

$$
\gamma_{\mathrm{D}} \simeq-\frac{1}{2}\left(\frac{\partial \ln B}{\partial \ln V}\right)_{T}-\frac{1}{4}
$$

The vibrational mode expressed by the Debye characteristic frequency of polyethylene, $\nu_{D}$, is considered to be closely related to the intermolecular mode, as suggested from the results of the analysis of the low-temperature specific heat of polyethylene crystals. $^{16}$ Thus, the Grüneisen constant given by eq 2 or 8 is considered to express mainly the anharmonicity of the intermolecular vibrations of polyethylene molecules in the crystal.

\section{Comparison of Grüneisen Values Obtained at 1} atm with Theoretical Ones

Curves 1 and 2 in Figure 6 show the temperature dependence of $\gamma_{\mathrm{S}}$ at $1 \mathrm{~atm}$ obtained by using eq 2 with the velocity data on polyethylene. Curve 1 is for the single crystal mats obtained by us (Figure 5) and curve 2 is for the bulk samples reported by Wada, et al. ${ }^{6}$ Considering the differences in experimental conditions and samples used between both authors, it may be said that curves 1 and 2 are in fairly good agreement. Thus, it is concluded that the Grüneisen constant, $\gamma_{\mathrm{s}}$, of polyethylene crystal at $1 \mathrm{~atm}$ increases gradually with increasing temperature below room temperature and its slope becomes steeper at higher temperatures.

The theoretical calculations of the Grüneisen constant of $n$-paraffine were carried out on the basis of eq 8 by Broadhurst and Mopsik., According to them, the Grüneisen constant of a linear polymer crystal was derived as a function of volume by assuming that (1) the bulk modulus or the Grüneisen constant can be described in terms of a bundle-like crystal model composed of rod-like chains, (2) the contribution of thermal pressure to the bulk modulus or the

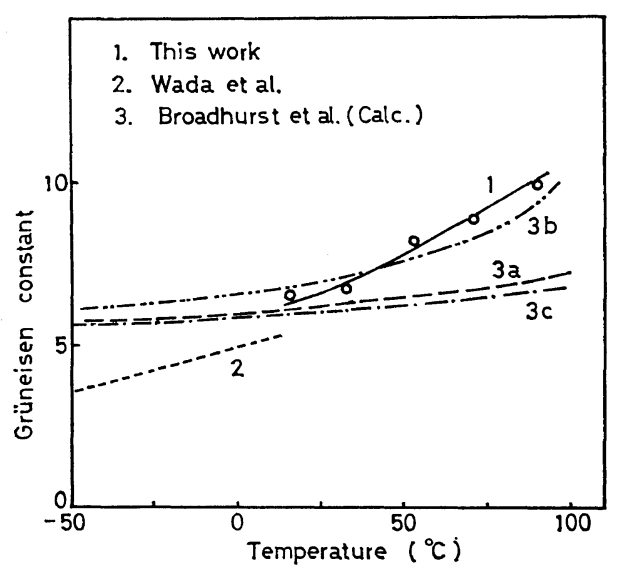

Figure 6. Temperature dependence of Grüneisen constant of polyethylene at $1 \mathrm{~atm}$ : (1) Experimentally determined $\gamma$ s (this work); (2) ibd. (Wada, et al. ${ }^{6}$ ); (3a) $\gamma_{\mathrm{L}}$ of perfect crystals obtained by extrapolating the theoretical $\gamma_{\mathrm{L}}$ of $n$-alkanes from Broadhurst, et al.; (3b) $\gamma_{\mathrm{L}}$ for the imperfect crystals with defects of $1 \%$ in volume, ibd.; (3c) $\gamma_{L}$ calculated using eq 9 and the $V-T$ data from Swan. ${ }^{17}$

Grüneisen constant associated with it is negligible, and (3) the pair potential for the isolated chains can be expressed by the Mie 6-12 potential, and besides by neglecting the second term on the right side of eq 8 . Thus, they obtained eq $9 .{ }^{4}$

$$
\gamma_{\mathrm{L}}=\frac{91-28\left(V / V_{0}\right)^{3}}{28-16\left(V / V_{0}\right)^{3}}
$$

where $V_{0}$ and $V$ are the volume of a perfect crystal at $0^{\circ} \mathrm{K}$ and that of a perfect or imperfect crystal at a temperature $\left({ }^{\circ} \mathrm{K}\right)$, respectively, and the suffix " $L$ " is used to denote that it is concerned only with lattice potential. It should be noticed that $\gamma_{\mathrm{L}}$ is implicitly related to temperature through the temperature dependence of the volume $V$ in eq 9. Furthermore, Broadhurst, et al., ${ }^{7}$ replaced their previous model mentioned above by a two-dimensional hexagonal lattice containing semirigid chains composed of $N$ beads, and obtained the $\gamma_{\mathrm{L}}$ value as a function of temperature, $T$, and the number of carbon atoms per chain, $N(N \leq 76)$, from the relation of eq 9 by using the volume evaluated on the basis of a quasiharmonic potential approximation. 
The long period of the single crystal mats used in the present work, $135 \AA$, is equivalent to $N=109$. Curves $3 \mathrm{a}$ and $3 \mathrm{~b}$ in Figure 6 show the temperature dependence of the $\gamma_{L}$ values of a perfect crystal and that of a imperfect one with defects of $1 \%$ in volume, respectively, which were obtained by extrapolating the $\gamma_{\mathrm{L}}$ value vs. $N$ plot to $N=109$. Curve $3 c$ shows the temperature dependence of the $\gamma_{\mathrm{L}}$ value which was obtained semiempirically from eq 9 using the data on the $V-T$ relation of polyethylene crystal reported by Swan. ${ }^{17}$

Now let us compare the temperature dependence of $\gamma_{\mathrm{s}}$ (curves 1 and 2) with those obtained theoretically (curves $3 a$ and $3 b$ ) and semiempirically (curve $3 \mathrm{c}$ ). In the temperature region below room temperature the experimental values of $\gamma_{\mathrm{s}}$ are smaller than any of the others. On the other hand, the theoretical value of $\gamma_{L}$ for a simple cubic crystal lattice model using the same potential as used in eq 9 is equal to 3.5 at equlibrium state, i.e., at $0^{\circ} \mathrm{K}$, which is very close to the obserbed value of $\gamma_{\mathrm{s}}$ near $-50^{\circ} \mathrm{C}$ (see curve 2). Therefore, the disagreement between curve 2 and the other ones below room temperature is probably due to the fact that at these low temperatures the crystal lattice expands rather three-dimensionally and thus the assumption of two-dimensional expansion of the crystal lattice seems to become invalid, as suggested by the strong anisotropy of the lattice expansion. ${ }^{17}$

The experimental values of $\gamma_{\mathrm{s}}$ above room temperature (curve 1) show larger values than the theoretical ones of $\gamma_{L}$ for the perfect crystals (curve 3a), but are nearly in accord with those for the imperfect crystals including defects of $1 \%$ in volume (curve $3 b$ ). This fact suggests that the experimental values of $\gamma_{\mathrm{S}}$ for the single crystal mats contain not only the contribution from the intermolecular vibrations but also that from the defects which seem to be present in the crystal lattice and in the interlamellar region.

\section{Comparison between The Grüneisen Constant and the X-Ray Temperature Factor}

Anharmonicity of molecular motions in the crystal lattice can be expressed not only by the Grüneisen constant, as mentioned above, but also more directly by using the symmetric tensor
$\mathbf{U}$ which denotes the mean square displacements of atoms in the crystal lattice and is related to the X-ray temperature factor $\mathbf{B}$ as follows.

$$
\mathbf{B}=4 \pi^{2} \mathbf{U}=8 \pi^{2}\left(\begin{array}{ccc}
U_{a a} & U_{a b} & U_{a c} \\
& U_{b b} & U_{b c} \\
& & U_{c c}
\end{array}\right)
$$

where subscripts of $a, b$, and $c$ denote the $a, b$, and $c$ axes of unit cell of polyethylene and their directions. The tensor $\mathbf{U}$ can be seperated into two terms as follows.

$$
\mathbf{U}=\mathbf{U}^{T}+\mathbf{U}^{0}
$$

where $\mathbf{U}^{T}$ and $\mathbf{U}^{0}$ express the contribution from the thermal vibrations of the molecules and that from the lattice imperfections, respectively. $U_{a a}^{T}, U_{b b}^{T}$, and $U_{c c}^{T}$ indicate the mean-square amplitudes of thermal vibrations of carbon atoms along the $a, b$, and $c$ axes, respectively. The relationship between the Grüneisen constant and the anharmonicity of the molecular motions in the crystal lattice might be calculated by referring to the information on mean-square displacements of carbon atoms of polyethylene molecules in the crystal obtained by X-ray measurements by Iohara, et al. ${ }^{8}$

Iohara, et al. ${ }^{8}$ have obtained the elements of tensor $\mathbf{U}$ for the polyethylene crystal by precise analysis of the X-ray diffraction at various temperatures under $1 \mathrm{~atm}$ and then determined the values of $\mathbf{U}^{T}$ in eq 11 . On the assumption that the tensor $\mathbf{U}^{0}$ can be evaluated by extrapolating the $U$ values to absolute zero along the temperature axis and substracting $\mathbf{U}^{0}$ from $\mathbf{U}$ to obtain the value of $\mathbf{U}^{T}$, they compared the values of $\mathbf{U}^{T}$ with the theoretical ones which had been calculated on the basis of a harmonic oscillator model by Kitagawa and Miyazawa. ${ }^{18}$ They found good agreement between the elements of the tensor $\mathbf{U}^{T}$ obtained by the X-ray measurements and the theoretical calculations in the low-temperature region below $0^{\circ} \mathrm{C}$, and concluded that the polyethylene molecules in the crystal lattice vibrate almost harmonically at these low temperatures. This supports the explanation that the larger values of $\gamma_{\mathrm{S}}$ with increasing temperature below $0^{\circ} \mathrm{C}$ (curve 2 in Figure 6) can be ascribed mainly to an increase in amplitude of the harmonic or quasiharmonic vibrations of the molecules in the crystal lattice. 
Iohara, et al. ${ }^{8}$ further confirmed that, in the temperature region above $0^{\circ} \mathrm{C}$, the values of $U_{a a}^{T}$ and $U_{b b}^{T}$ increase more rapidly with increasing temperature than at the lower temperatures, and that the $U_{c c}^{T}$ values increase rapidly above $50^{\circ} \mathrm{C}$. In such a temperature region, all of the values of experimental $U_{i i}$ show larger values than those of the theoretical $U_{i i}^{T}$ values obtained on the assumption of a harmonic oscillator model. Thus, the increase of $\gamma_{\mathrm{s}}$ above room temperature as shown by curve 1 in Figure 6 seems to be ascribable to the increase of anharmonicity of the translational and rotational modes of motions in the plane perpendicular to the molecular axis, as well as to the increase of molecular displacements due to the lattice imperfections as inferred by the overlapping of curves 1 and $3 b$ in the higher temperature region in Figure 6. Above about $50^{\circ} \mathrm{C}$ the increase of anharmonicity of the translational vibrations along the molecular axis may also contribute considerably to the increase in the Grüneisen constant.

\section{Pressure Effect on the Grüneisen Constant}

In the last section our attention was focussed on the Grüneisen constant at $1 \mathrm{~atm}$. The Grüneisen constant, $\gamma_{s}$, at higher pressures shows a smaller dependence on temperature than that at $1 \mathrm{~atm}$, as shown in Figure 5. This fact suggests that the molecular displacements in the crystal decrease with increasing pressure owing to the decrease in the volume of lattice imperfections and also in the anharmonicity of intermolecular vibrations which are probably accelerated by compression of the crystal lattice. The latter contribution might arise from the nature in the anharmonicity of intermolecular potential similar to that of the so-called Lennard-Jones potential for a pair of atoms, as the anharmonicity of the former potential at intermolecular distances greater than the equilibrium value of it decreases with a decrease of intermolecular distance. Furthermore, the decrease of anharmonicity of intermolecular vibrations with increasing pressure is qualitatively consistent with the fact that the temperature location of the $\alpha_{2}$ mechanical relaxation which is probably caused by the increase of intermolecular vibrations in the crystal lattice ${ }^{19}$ shifts to the higher temperature side with increasing pressure. $^{20}$

Acknowledgment. The authors wish to thank Professor T. Takemura and Mr. S. Taki in Department of Applied Science of Kyushu University for their helpful technical advice in the ultrasonic and high pressure measurements.

\section{REFERENCES}

1. C. K. Wu, G. Jura, and M. Shen, J. Appl. Phys., 43, 4348 (1972).

2. R. E. Barker and R. Y.S. Chen, J. Chem. Phys., 53, 2615 (1970).

3. C. K. Wu and M. Nicol, Bull. Amer. Phys. Soc., 17, 274 (1972).

4. M. G. Broadhurst and F. I. Mopsik, J. Chem. Phys., 52, 3634 (1970).

5. T. Ito and H. Marui, Polymer J., 2, 768 (1971).

6. Y. Wada, A. Itani, T. Nishi, and S. Nagai, $J$. Polym. Sci., Part A-2, 7, 201 (1969).

7. M. G. Broadhurst and F. I. Mopsik, J. Chem. Phys., 54, 4239 (1971).

8. K. Iohara, K. Imada, and M. Takayanagi, Polymer J., 3, 357 (1972).

9. W. R. D. Manning, Engineering, 163, 349 (1947).

10. S. Hirakawa and T. Takemura, Jap. J. Appl. Phys., 7, 814 (1968).

11. R. W. Warfield, J. Appl. Chem., 17, 263 (1967).

12. K. H. Hellwege, W. Knappe and P. Lehman, Kollid-Z., 183, 110 (1961).

13. J. C. Slater, "Introduction to Chemical Physics," McGraw-Hill Book Inc., 1939, Chapter 13.

14. C. Kittel, "Introduction to Solid State Physics," John Willy \& Sons, Inc., New York, N.Y., 1966, Chapter 6.

15. J. S. Dugdale and D. K. C. MacDonald, Phys. Rev., 89, 832 (1953).

16. B. Wunderlich, J. Chem. Phys., 37, 1207 (1962).

17. R. P. Swan, J. Polym. Sci., 52, 525 (1960).

18. T. Kitagawa and T. Miyazawa, Advans. Polym. Sci., 9, 336 (1972).

19. K. Okano, J. Polym. Sci., Part C, 15, 95 (1966).

20. T. Kijima, K. Koga, K. Imada, and $\mathbf{M}$. Takayanagi, to be published. 